\title{
Wärmeübergang bei der Verdampfung der verflüssigten Gase Helium und Stickstoff
}

\section{Doctoral Thesis}

Author(s):

Frederking, Traugott

Publication date:

1961

Permanent link:

https://doi.org/10.3929/ethz-a-000099252

Rights / license:

In Copyright - Non-Commercial Use Permitted 
Prom. Nr. 3007

\title{
WÄRMEÜBERGANG BEI DER VERDAMPFUNG DER VERFLÜSSIGTEN GASE HELIUM UND STICKSTOFF
}

\author{
Von der \\ EIDGENÖSSISCHEN TECHNISCHEN HOCHSCHULE \\ IN ZÜRICH \\ zur Erlangung der Würde eines Doktors \\ der technischen Wissenschaften \\ genehmigte

\section{PROMOTION SARB EIT} \\ Vorgelegt von \\ TRAUGOTT FREDERKING \\ Dipl.-Ing. \\ Deutscher Staatsangehöriger
}

Referent: Herr Prof. Dr. P. Graßmann

Korreferent: Hert Prof. M. Berchtold 


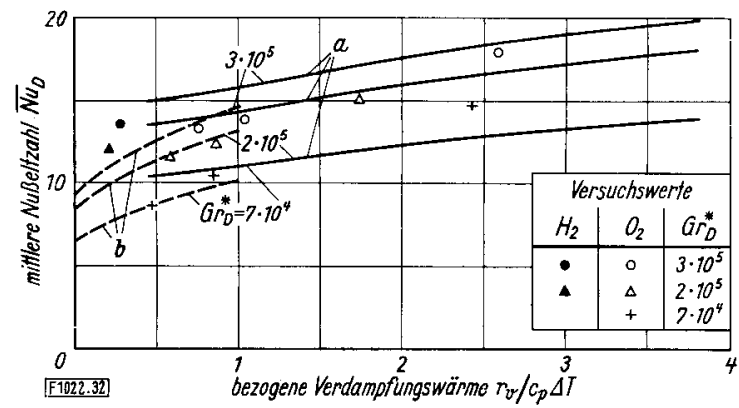

Bild 32. Vergleich von Theorie und Versuch bei großen Grashofzahlen. a und $b$ theoretischer Verlauf nach Gl. (37) bzw. nach Gl. (38)

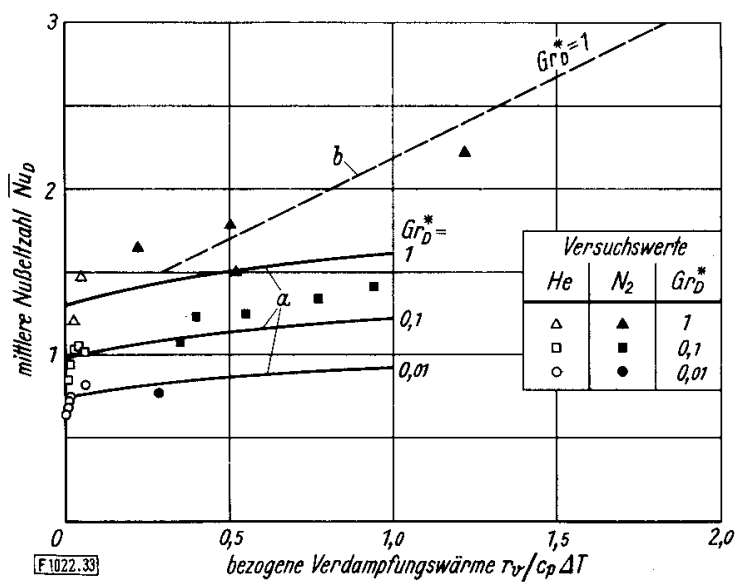

Bild 33. Vergleich von Theorie und Versuch bei kleinen Grashofzahlen. a Verlauf nach Gl. (49), b auf $\operatorname{Gr}_{D}^{*}=1$ umgerechnetes Meßergebnis für einen Draht von $16,2 \mu \mathrm{Dm}$.

Bild 32 und 33. Einfluß der durch die bezogene Verdampfungs wärme $r_{v} / c_{p} \Delta T$ gekennzeichneten Filmüberhitzung auf die mittlere Nußeltzahl $\overline{\mathrm{Nu}}_{D}$ an waagerechten Zylindern bei verschiedenen Werten der modifizierten Grashofzahl $\mathrm{Gr}_{D}^{*}$.

Das dimensionslose Gesetz der Filmverdampfung an waagerechten Zylindern (vgl. Bild 31) hat bei hoher Filmüberhitzung große Ähnlichkeit mit der für natürliche Konvektion annähernd gültigen Abhängigkeit der Nußeltzahl als Funktion des Produktes der Grashof- und der Prandtlzahl. Es liegt deshalb nahe, Rechnungsverfahren zum Ermitteln der Wärmeabgabe dünner Drähte bei natürlicher Konvektion auch auf das Filmsieden bei hoher Úberhitzung anzuwenden. Erwähnt sei nur die Gleichung von $H$. Senftleben [53], deren numerische Werte wenig von den neuen Meßwerten der Nußeltzahl abweichen. Bei Analogieschlüssen sollte man jedoch die unterschied- lichen Strömungsformen, die besonders beim Bilden von Einzelblasen entstehen, berücksichtigen. Am Rande sei bemerkt, daß für hohe Filmüberhitzung die Messungen an dünnen Drähten näherungsweise auch durch Gleichungen von der Form

$$
\overline{\mathrm{Nu}}_{D}=C(\mathrm{Gr} \operatorname{Pr})^{m_{1}}
$$

mit $C$ und $m_{1}$ als zwei Konstanten sowie mit $\mathrm{Gr}=$ $D^{3} g \Delta T / T v^{2}$ wiedergegeben werden können [54] (Stoffgrößen bei arithmetischer Mitteltemperatur). Dann ist jedoch die Konstante $C$ eine Funktion von $p / p_{\mathrm{c}}$ mit $p_{\mathrm{c}}$ als dem kritischen Druck. Für Helium ist $C \approx 2,5$ und $m_{1} \approx 0,11$ in einem Bereich des Produktes Gr Pr von $10^{-7}$ bis $10^{-1}$ bei $p=1$ atm.

\section{Zusammenfassung}

Es wurde der Wärmeübergang bei der Verdampfung von Helium und Stickstoff an dünnen elektrisch geheizten Drähten (Durchmesser $D=5$ bis $200 \mu$ ) gemessen. Bei der Blasenverdampfung in Stickstoff ist der Wärmeübergang an dünnen Drähten ähnlich hoch wie bei großen Heizelementen, solange eine intensive Blasenbildung stattfindet. An sehr dünnen Drähten $(D<\approx 30 \mu)$ in Stickstoff kann die Blasenentwicklung sehr zurückgehen; trotzdem bleiben die Wärmestromdichten groß (5 bis $10 \mathrm{~W} / \mathrm{cm}^{2}$ ), da der durch natürliche Konvektion bedingte Wärmeübergang am dünnen Draht ohnehin hoch ist. Bei Drücken unter 1 atm läßt sich bei Helium und Stickstoff die Abhängigkeit des maximalen Wärmeflusses der Blasenverdampfung vom Druck in erster Näherung durch Beziehungen nach der Art von Gl. (2) wiedergeben. Dio gemessenen Werte der maximalen Wärmestromdichten weichen jedoch $u$. U. erheblich von den theoretischen Werten ab (sie lagen z. B. beim waagerechten Draht mit $D=15 \mu$ in Helium mehr als doppelt so hoch). Bei He II ist der maximale Wärmefluß vor dem Umschlag auf Filmverdampfung mit $10 \mathrm{~W} / \mathrm{cm}^{2}$ um eine Größenordnung höher als bei He I.

Bei der Filmverdampfung am waagerechten Zylinder mit laminar strömendem Film hängt nach den experimentellen und theoretischen Befunden die Nußeltzahl von der mit der Dichte gebildeten modifizierten Grashofzahl $\mathbf{G r}_{D}^{*}$, der Dimensionslosen $r_{\mathrm{v}} / c_{p} \Delta T$ und der Prandtlzahl Pr ab. Die Frgebnisse der Messungen an dünnen Drähten in Helium und Stickstoff lassen sich ebenso wie aus dem Schrifttum entnommene Daten für Drähte und Rohre in verflüssigten Gasen bei großer Úberhitzung des Gases im Film e inheit. lich darstellen, obwohl sich bei Stickstoff der Film in Einzelblasen auflöst. Für den Bereich $r_{\mathrm{v}} / c_{p} \Delta T<0,9$ ergibt sich die Nußeltzahl angenähert nach Gl. (49).

Bei sehr kleinem $r_{\mathrm{v}} / c_{p} \Delta T$, d. h. bei großer Filmüberhitzung, wie sie ohne Strahlungseinfluß in Helium möglich
Bild 34. Abhängigkeit der mittleren NuBeltzahl $\overline{N u}_{D}$ von der Funktion $\operatorname{Gr}_{D}^{*} f$ für einen Draht von 16,2 $\mu \mathrm{Dmr}$. in Stickstoff.

a Verlauf nach Gl. (49) für $p=1$ atm

Bild 34 und 35. Wärmeübergang bei Filmverdampfung unter niedrigem Druck $p$.

Bild 35. Abhängigkeit der mittleren Nußeltzahl $\overline{\mathrm{Nu}}_{D}$ von der Funktion $\mathrm{Gr}_{D}^{*}$ für einen Draht von $16,2 \mu$ Dmr. in Helium. a Verlauf nach Gl. (48) für $p=1 \mathrm{~atm}$
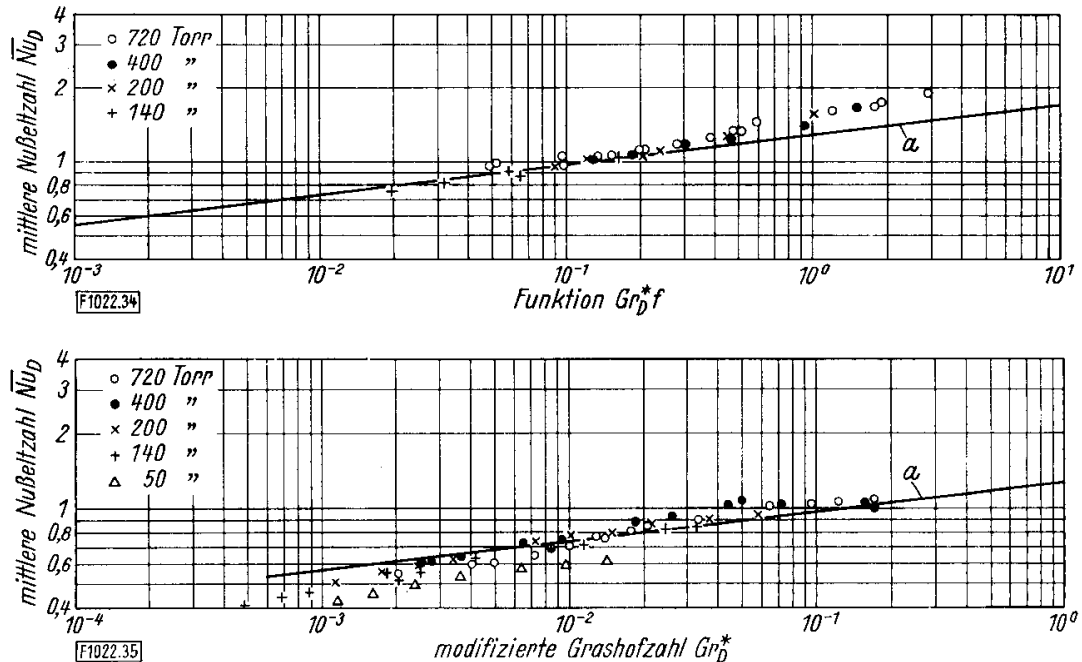
ist, hängt die Nußeltzahl in ganz ähnlicher Weise wie bei der natürlichen Konvektion o h n e Verdampfung nur noch vom Produkt der Grashof- und der Prandtlzahl ab. Unter bestimmten Bedingungen kann der Wärmeübergang von dem durch die angegebene Gleichung festgelegten Verlauf abweichen. So wird bei großen mittleren freien Weglängen des Gases im Film die Nußeltzahl vermindert, bei hohen Temperaturdifferenzen hingegen durch den Strahlungseinflu $\beta$ erhöht. Auch infolge von turbulenter Strömung an lotrechten dicken Rohren ergeben sich höhere Nußeltzahlen. Im Gegensatz dazu ist der Wärmeübergang am lotrechten dünnen Draht in Helium und Stickstoff annähernd ebenso groß wie am waagerechten Draht vom gleichen Durchmesser. Bei der Filmverdampfung in Helium weist die Wärmeübergangszahl beim Übergang von He I zu He II am $\lambda$-Punkt keinen Sprung auf, obwohl sich dort die Flüssigkeitseigenschaften in ungewöhnlicher Weise ändern.

\section{Sehrifttum}

[1] Roubeau, P.: Heat exchanges in nitrogen and hydrogen boiling under pressure. $10^{\circ}$ Congr. Internat. du Froid, Kopenhagen 1959. Bull. Inst. Internat. Froid 39 (1959) Nr. 3 S. 746.

[2] Eckeri, E.: Einführung in den Wärme- und Stoffaustausch. 2. Aufl. Berlin/Göttingen/Heidelberg 1959.

[3] Bonilla, C. F.: Nuclear engineering. New York/Toronto/ London 1957 ; insbes. S. $360 / 518$

[4] Westwater, J.W.: Boiling of liquids. In: Drew, T. B., u. $J . W$. Hoopes: Advances in chemical engineering. Bd. 1. New York 1956; insbes. S. $1 / 76$ u. Bd. 2. New York 1958; insbes. S. $1 / 31$

[5] Hoare, F. E., u. J. E. Zimmermann: Helium temperatures from vapor pressure measurements. Rev. sci. instr. 39 (1959) Nr. 3 S. 184/86.

[6] van Dijk, H., u. M. Durieux: Thermodynamic temperature scale $\left(T_{L_{55}}\right)$ in the liquid helium region. Physica 24 (1958) Nr. 1 S. 1119.

[7] Armstrong, G. T.: Vapor pressure of nitrogen. J. Res. Nat. Bur. Stand. 53 (1954) Nr. 4 S. 263/66.

[8] Carslaw, H. S., u. J. C. Jaeger: Conduction of heat in solids. 2. Aufl. Oxford 1959 ; insbes. S. 152.

[9] Preckshot, G.W., u. J.W. Gorman: Steady-state longitudinal and radial temperature distributions in internally heated finite wires. Industr. Engng. Chem. 50 (1958) Nr. 5 S. $837 / 48$.

[10] McAdams, W. H., J. N. Addoms, P. M. Rinaldo u. R. S. Day: Heat transfer from single horizontal wires to boiling water. Chem. Engng. Progr. 44 (1948) Nr. 8 S. 639/46.

[11] Lowenthal, G. C., W. R. G. Kemp u. A. F. A. Harper: A temperature scale down to $20^{\circ} \mathrm{K}$ using platinum resistance thermometers. Suppl. Bull. Inst. Internat. du Froid, Annexe 1 (1958) S. 107/16.

[12] White, G. K.: Experimental techniques in low-temperature physics. Oxford 1959; insbes. S. 115. Vgl. auch: American Institute of Physics Handbook. New York/Toronto/Lond on 1957 ; insbes. S. $4 / 13$

[13] Corty, C., u. A. S. Foust: Surface variables in nucleate boiling. Chem. Engng. Progr. Symposium Series 51 (1955) Nr. 17 S. $1 / 12$

[14] Weil, L.: Échanges thermiques dans les liquides bouillants. IVe Congrès International du Chauffage Industriel. Paris 1952, Bericht Nr. 210

[15] Weil, L., u. A. Lacaze: Coefficients d'échange thermique dans l'azote bouillant. Compt. Rend. $\mathbf{2 3 0}$ (1950) S. 186/88.

16] Weil, L., u. A. Lacaze: Variation avec la pression des coefficients d'échange dans l'azote bouillant. J. Phys. Rad. 12 (1951) S. $45 \mathrm{~S} / 46 \mathrm{~S}$.

[17] Ružička, J.: Heat transfer to boiling nitrogen. Suppl. Bull. Inst. Internat. du Froid, Annexe 1 (1958) S. 323/29.

[18] Mesler, R. B., u. J. T. Banchero: Effect of superatmospheric pressures on nucleate boiling of organic liquids. A.I.Ch.E. J. 4 (1958) Nr. 1 S. 102/13.

[19] Mulford, $R . N$., u. J. P. Nigon: Heat exchange between a copper surface and liquid hydrogen and nitrogen. Los Alamos Scientific Laboratory of the University of California, Rep. IAA-1416 (1952).
[20] Kutateladse, S. S.: A hydrodynamic theory of changes in the boiling process under free convection conditions. Izv. Akad. Nauk., USSR, Otd. Tekh. Nauk 4 (1951) S. 529.

[21] Keesom, W. H., u. A. Bijl: Determination of the vapor pressures of liquid nitrogen below one atmosphere and of solid nitrogen $\beta$. Physica 4 (1937) Nr. 4 S. 305/10.

[22] Borishanskii, V.M.: An equation generalizing experimental data on the cessation of bubble boiling in a large volume of liquid. Zhurn. Tekh. Fiz. 26 (1956) S. 452; übersetzt in Soviet Physics-Technical Physics 1 (1956) S. 438/42, (veröff. vom American Institute of Physics, New York, N.Y.).

[23] Karagounis, A.: Heat transfer coefficient for liquid helium. Suppl. Bull. Inst. Internat. du Froid, Annexe 2 (1956) S. $195 / 99$.

[24] Grassmann, P., A. Karagounis, J. Kopp u. T. Frederking: Wärmeübergang an flüssiges Helium bei Blasen- und stabiler Filmverdampfung. Kältetechnik 10 (1958) $\mathrm{Nr} .7$ S. 206/08.

[25] Meissner, H.: Paramagnetic effect in superconductors. V. Resistance transition of tin wires. $X$. Heat transfer from horizontal wires to the liquid helium. Phys. Review 109 (1958) Nr. 3 S. 677/79.

[26]Andronikashvili, E. L., u. G. G. Mirskaia: The behaviour of helium II in the neighbourhood of a heat radiating surface. Soviet Physics - Technical Physics 2 (1956) S. $406 / 09$.

[27] Harrison, W. B., u. Z. Levine: Wetting effects on boiling heat transfer: the copper-stearic acid system. A.I.Ch.E. J. 4 (1958) Nr. 4 S. 409/12.

[28] Griffith, P.: Bubble growth rates in boiling. Trans. Amer. Soc. mech. Engrs. 80 (1958) S. 721/27.

[29] Forster, H. K., u. N. Zuber: Growth of a vapor bubble in a superheated liquid. J. appl. Phys. 25 (1954) Nr. 4 S. 474/78.

[30] Faneuff, C. E., E. A. McLean u. V. E. Scherrer: Some aspects of surface boiling. J. appl. Phys. 29 (1958) Nr. 1 S. $80 / 84$.

[31] Zuber, N.: On the stability of boiling heat transfer. Trans. Amer. Soc. mech. Engrs. 80 (1958) S. 711/20.

[32] Grassmann, P.: Eigenschaften des flüssigen und festen Heliums. Vierteljahrsschrift der Naturforschenden Gesellschaft Zürich 102 (1957) Nr. 3 S. $61 / 87$.

[33] Atkins, K. R.: Liquid helium. Cambridge 1959.

[34] Lifshits, E. M., u. E. L. Andronikashvili: A supplement to "Helium". New York 1959; insbes. S. 131.

[35] Rinderer, L., u. F. Haenssler: Transfer de chaleur dans l'hélium superfluide. Helv. Phys. Acta 32 (1959) Nr. 4 S. 322/25.

[36] Keesom, W. H., B. F. Saris u. L. Meyer: New measure. ments on the heat conductivity of liquid helium II. Physica 7 (1940) Nr. 9 S. $817 / 30$

[37] Kapitza, P. L.: The study of heat transfer in helium II. J. Phys. (USSR) 4 (1941) Nr. 3 S. 181/210.

[38] Westwater, $J . W .$, u. J.G. Santangelo: Photographic study of boiling. Industr. Engng. Chem. 47 (1955) Nr. 8 S. 1605/10

[39] Grossmann, U.: Stoff- und Wärmeaustausch zwischen Flüssigkeit und aufsteigenden Dampfblasen bei Zweistoffgemischen. Diss. Eidgen. Techn. Hochschule Zürich, Prom.-Nr. 2396 (1955); vgl. a. Grossmann, U.: Untersuchung über den Stoff- und Wärmeaustausch zwischen Flüssigkeit und Dampfblasen bei Alkohol-WasserGemischen. Chemie-Ing.-Techn. 28 (1956) Nr. 2 S. 107/12.

[40] Frank, A.: Wärme- und Stoffaustausch zwischen Dampfblase und Flüssigkeit bei $\mathrm{N}_{2}-\mathrm{O}_{2}$-Gemischen. Diss. Eidgen. Techn. Hochschule Zürich, Prom.-Nr. 2827 (1958).

[41] Clusius, K., C. G. Losa u. P. Franzosini: Ergebnisse der Tieftemperaturforschung. XVIII: Die Atom- und Elektronenwärme des Platins zwischen 10 und $273^{\circ} \mathrm{K}$. Z Naturforsch. 12a (1957) Nr. 1 S. 34/38; vgl. a.: American Institute of Physics Handbook. New York / Toronto / London 1957 insbes. S. $4 / 43$

[42] Stenzel, W., u. G. R. Schultze: Verdampfungskühlung. Chemie-Ing.-Techn. 30 (1958) Nr. 10 S. 643/47.

[43] Schlichting, H.: Grenzschicht-Theorie. 3. Aufl. Karlsruhe 1958; insbes. S. 219/45.

[44] Howarth, L.: Laminar boundary layers. In: Flügge, S.: Handbuch der Physik. Band VIII/1: Strömungsmechanik I Berlin/Göttingen/Heidelberg 1959; insbes. S. 264/350.

[45] Goldstein, S.: Modern developments in fluid dynamics. Bd. 2. Oxford 1938; insbes. S. $641 / 43$.

[46] Nußelt, W.: Die Oberflächenkondensation des Wasser- 